\title{
Cefazolin associated with better outcomes than is vancomycin for MSSA infection
}

A new study indicates that although vancomycin is the most commonly used antibiotic for patients with methicillinsensitive Staphylococcus aureus (MSSA) bacteremia, the $\beta$-lactam antibiotic cefazolin may be more effective than vancomycin at reducing the risk of hospitalization and death.

Patients on haemodialysis are at high risk of bloodstream infection. "According to the 2011 United States Renal Data System Annual Report, death from infection occurs at a rate of 23.1 per 1,000 patientyears in the haemodialysis population", say investigators Dr Kevin Chan and Dr Franklin Maddux. "Hospital admissions for infection are also on the rise, reaching a rate of 471 per 1,000 patient-years in 2011. Consequently, there is a need to identify opportunities to reduce infection-related morbidity and mortality in the population with end-stage renal disease."

In order to study trends in vancomycin and cefazolin prescription and the associated use of each antibiotic with subsequent hospitalization or death related to infection, data from 293,094 haemodialysis outpatients over a 5-year period were analysed. The bloodstream infection rate was 2.1 per 100 outpatientyears for MSSA and 1.9 per 100 outpatientyears for methicillin-resistant $S$. aureus (MRSA) bacteremia. 1 week after blood specimens were collected, $74.7 \%$ of outpatients with MRSA and 56.1\% of outpatients with MSSA were treated with vancomycin. Only $16.7 \%$ of outpatients with MSSA were treated with cefazolin and use of cefazolin for MSSA bacteremia decreased by $8 \%$ from 2006 to 2010 whereas vancomycin use increased. Compared with vancomycin, cefazolin was associated with a significant 38\% lower combined risk of hospitalization or death from infection in MSSA bacteremia and a significant $48 \%$ reduction in the rate of sepsis. In sensitivity analyses, cefazolin was still associated with reduced hospitalization and death from infection compared with vancomycin. However, in patients with MRSA bacteremia, the best outcomes were associated with vancomycin.

"The data suggest there is opportunity to improve outcomes for patients through

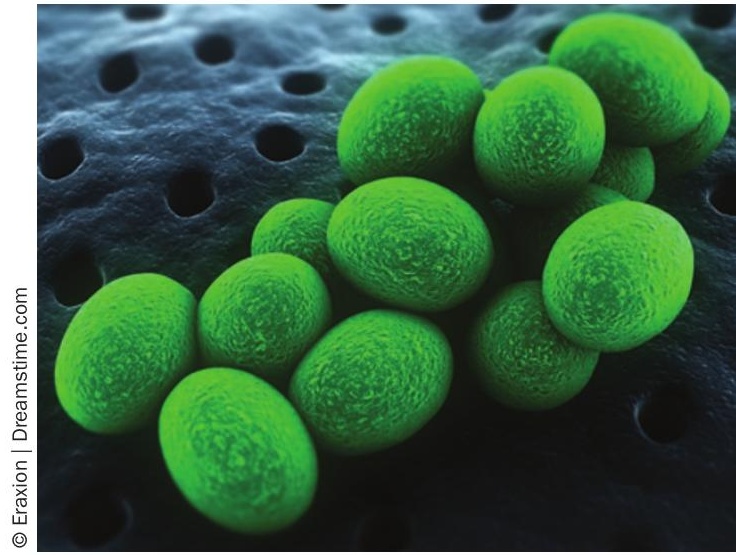

appropriate antibiotic selection", say Chan and Maddux. "Perhaps our study will help facilitate improved processes of care in bloodstream infection management."

Helene Myrvang

Original article Chan, K. E. et al. Prevalence and outcomes of antimicrobial treatment for Staphylococcus aureus bacteremia in outpatients with ESRD. J. Am. Soc. Nephrol. doi:10.1681/ASN.2012010050 